\title{
Über den Befruchtungsvorgang bei der Miesmuschel (Mytilus edulis L.).
}

\author{
Von \\ Friedrich Meves in Kiel.
}

Hierzu Tafel V.

Die Auffassung von dem Wesen der Befruchtung hat auch nach Entdeckung der "Samenkörperchen" (1677) gemäss dem jeweiligen Zustand der wissenschaftlichen Erkenntnis stark gewechselt. Nachdem die Theorie Leeuwenhooks, welcher die Samenkörperchen als die präexistierenden Keime ansah, sich als irrtümlich herausgestellt hatte, wurden sie lange Zeit von den "berühmtesten und, was mehr sagt, den berechtigten Autoritäten “ für parasitische Infusorien des Samens gehalten. „Nur dadurch“, sagt Hensen 1885, S. 739, „dass trotz allen Glaubens an jene Autoritäten das Studium der Natur fortgesetzt wurde, sind wir weiter gekommen, denn die Natur fahrt fort, ihre unwandelbaren Tatsachen zu geben, vor denendie Irrtümer jeder Autorität zerstäuben“. Kölliker (1841) war der erste, der die Lehre von der selbständigen tierischen Natur der "Spermatozoiden" mit Entschiedenheit bekămpfte und zeigte, dass sie Produkte des văterlichen Organismus sind. Schon vorher war durch Spallanzani (1785) und Prévost und Dumas (1824) auf experimentellem Wege nachgewiesen worden, dass die Samenkörperchen zum Zweck der Befruchtung mit den Eiern in unmittelbare Berührung kommen müssen. Man sah sje dann auch (um 1850) den Eizellen ăusserlich anhängen, später aber verloren gehen. Die Frage nach ihrer Wirkungsweise beantwortete man damals der Hauptsache nach folgendermaßen: Nach einer Ansicht sollten sie sich auflösen und ihre Substanz. der Dottermasse zumischen; die Befruchtung sollte also durch. von aussen eindringende gelöste Stoffe erfolgen. Eine andere Anschauung, welche von Bisch off unter dem Einfluss der Liebigschen Lehre von den Kontaktwirkungen aufgestellt und z. B. von Leuckart akzeptiert wurde, lautete dahin, dass es. sich bei der Befruchtung um die Mitteilung einer inneren Archiv 1. mikr. Anat. Bd. 87. Abt. $\mathrm{Ix}$. 
Molekularbewegung handle, die in dem einen Körper, dem Spermatozoiden, bereits vorhanden, auf das zu derselben Form der Bewegung sehr geneigte $\mathrm{Ei}$ übergehe. ${ }^{1}$ )

Später wurden von verschiedenen Seiten Beobachtungen publiziert, nach welchen die Spermatozoiden in die Eizellen eindringen. Die theoretische Auffassung der Befruchtung änderte sich aber erst, als 0 . Hertwig 187 beim Seeigel fand, dass der Kopf des eingedrungenen Samenfadens sich zu einem Kern, dem Samenkern, umwandelt und dass der letztere mit dem weiblichen oder Eikern kopuliert. O. Hertwig stellte daraufhin den Satz auf, dass "die Befruchtung auf der Verschmelzung von geschlechtlich differenzierten Zellkernen beruht". Hensen (1881, S. 126) bezeichnete diese Auffassung insofern als eine glückliche, als sie unsere Kenritnisse von dem Befruchtungsvorgang vertiefe, indem sie za den bisher nur in Betracht gezogenen chemischen und physikalischen Momenten noch hinzufüge das für die Lebenserscheinungen (und die Vererbung) so bedeutsame morphologische Moment, dass nämlich die Materie in bestimmter Formung mitwirkt". Van Beneden erbrachte sodann 1883 bei Ascaris den Nachweis, dass die Chromosomen der ersten Furchungsspindel zur einen Hălfte rom Eikern, zur anderen Hülfte vom Samenkern abstammen. Das Chromatin steht sich also im Ei- und Samenkern äquivalent gegenüber und wird auf die aus dem befruchteten $\mathrm{Ei}$ hervorgehenden Zéllen in gleicher Weise verteilt. Hierzu kommt, dass es vor der Befruchtung eine Massenreduktion erfährt. Auf Grund dieser Tatsachen gelangte die 0. HertwigStrasburgersche Lehre zur Herrschaft, dass das Chromatin als der alleinige Träger der erblichen Eigenschaften anzusehen sei.

Zwar wurde von Anfang an von den verschiedensten Autoren immer wieder darauf hingewiesen, dass das Spermium auch noch eine wenn auch nur sehr geringe Menge von Protoplasma mitbringt, welches zu vernachlässigen kein Grund vorliege. Die Anhänger der nuklearen Vererbungstheorie konnten sich jedoch bis vor kurzem mit Recht darauf berufen, dass diesen Hinweisen, soweit sie von deskriptiver Seite kamen, keine positiven Beob-

1) Für weiteres und die Literatur bis 1853 verweise ich auf den Artikel ron Leuckart: "Zeugung" in Wagners Handwörterbuch der Physiologie, Bd. 4. 
achtungen zugrunde lägen; die Ergebnisse der experimentellen Forschung aber, welche zu dem Kernmonopol der Vererbung in Widerspruch stehen, wurden von ihnen nicht als beweiskräftig anerkannt (vergl. Me ves 1908, S. 823).

In den letzten Jahren ist es nun aber mit Hilfe neuerer technischer Methoden gelungen, den Nachweis zu führen, dass bei der Befruchtung spezifische protoplasmatische Bestandteile, die Plastosomen, welche dem Ei und Spermium gemeinsam sind, von dem letzteren in das Eiprotoplasma übertreten. Angesichts der grossen Bedeutung, welche diesen Elementen für das zellulare Leben zugesprochen werden muss, ist es nicht anders glaublich, als dass sie bei der Übertragung erblicher Eigenschaften mitwirken. Die Lehre, nach welcher der Kern der alleinige Vererbungsträger ist, kann daher heute nicht mehr aufrecht erhalten werden.

Eine Aussaat männlicher Plastosomen im Eiprotoplasma ist bei Nematoden, bei welchen der Befruchtungsvorgang der Beobachtung besonders leicht zugänglich ist, bereits in zwei Fällen, bei Ascaris (L. und R. Zoja, Meves, Romeis, Held) und Filaria (Meves), nachgewiesen worden. Bei einer Ascidie, Phallusia, konnte i ch ferner an der röhrenförmigen plastosomatischen Scheide, welche bei diesem Tier den Kopf des Spermiums umgibt, bei der Befruchtung interessante Veränderungen wahrnehmen; ich fand, dass der Kopf sich auf einem späteren Stadium dieser Scheide entledigt hat, ohne dass es mir jedoch gelang, über ibr Schicksal etwas Bestimmtes festzustellen. Bei den grossen Schwierigkeiten der Untersuchung wird man sich überhaupt in vielen Fällen mit der Konstatierung begnügen müssen, dass bei der Befruchtung männliche plastosomatische Substanz mit dem Spermium in das Ei eingeführt wird. Über diesen Nachweis bin ich auch bei der vorliegenden Untersuchung, welche sich mit dem Befruchtungsvorgang bei der Miesmuschel (Mytilus edulis L.) beschäftigt, nicht hinausgelangt.

\section{Material und Methode.}

Die reifen Geschlechtsprodukte werden bei den Muscheln ebenso wie z. B. bei Echinodermen ins Wasser abgegeben. Wenn man in der geeigneten Jahreszeit eine Anzahl Miesmuscheln in Gläsern mit Seewasser isoliert, kann man, wie schon 0 . Hertwig 
(1877) mitteilt, bei einigen derselben die Entleerung ihrer Geschlechtsdrüsen in den nächsten Stunden beobachten und dann die künstliche Befruchtung ausfübren. Auf diese Weise konnte ich mir in Kiel in der Zeit von Ende April bis Anfang Juni ohne grosse Schwierigkeit Material verschaffen.

Von den verschiedenen, zum Studium der Plastosomen geeigneten Methoden, welche ich für die Untersuchung in Anwendung gebracht habe, hat mir die Altmannsche die besten Resultate gegeben, so dass ich mich schliesslich auf diese beschränkt habe. Der Farbung habe ich, wie auch früher, der Empfehlung von $\mathrm{Rubaschkin}$ folgend, eine Beizung nach Lustgarten-Pal vorausgehen lassen. Nachdem der Kopf des Samenfadens tiefer in das Ei eingedrungen ist, wird seine Auffindung durch zahlreiche im Ei vorhandene Fett-oder Dotterkügelchen erschwert, welche durch die in dem Altmannschen Gemisch enthaltene Osmiumsäure geschwärzt worden sind. Es empfiehlt sich daher, wenn andere als die allerersten Befruchtungsstadien zur Untersuchung kommen sollen, die aufgeklebten Schnitte zunăchst für 6-8 Stunden in Terpentin aufzustellen, welches die osmierten Kügelchen in Lösung bringt. Man kann sich den Nachweis des Spermienkopfes ferner dadurch erleichtern, dass man der Plastosomenfärbung mit Säurefuchsin-Pikrinsäure eine Kernfärbung mit Hämalaun vorausschickt. $\mathrm{Zu}$ diesem $\mathrm{Zweck}$ habe ich die Schnitte, nachdem ich sie zunächst nach Lustgarten-Pal gebeizt (eventuell auch noch vorher mit Terpentin behandelt) hatte, für ca. 12 Stunden in eine Hamalaunlösung nach $P$. Mayer hineingebracht, welche im Verhältnis 1:3 mit destilliertem Wasser verdünnt war.

\section{Die Spermien.}

$\mathrm{Zu}$ den überaus zahlreichen Spermienformen, deren genaue Kenntnis wir G. Retzi us verdanken, gehören auch diejenigen von Mytilus. Ich habe zu seiner Schilderung (1904, S. 22) nur weniges hinzuzusetzen.

„Der Kopf", sagt Retzius, „ist beinahe kuglig und im ganzen nicht gross, aber an seinem Vorderende findet sich ein mit breiter, von der Kopfsubstanz scharf abgesetzter Basis versehenes, nach vorn hin weit hervorragendes und stark zuge- 
spitztes Perforatorium, welches stärker lichtbrechend, glänzender als die Kopfsubstanz ist".

Die Abweichungen, welche der eigentliche Kopf von der Kugelform aufweist, bestehen nach $m$ e in e $n$ Beobachtungen darin, dass er etwas länger als breit und an seinem hinteren Umfang abgeplattet ist. Das Perforatorium ist in einigen meiner Präparate, welche nach der Altmann schen Methode hergestellt sind, durch und durch rot gefärbt. In anderen Präparaten hat es sich infolge stärkerer Differenzierung in seiner Achse aufgehellt; es erscheint also röhrenförmig. Die Wand der Röhre zeigt sich an der Basis etwas verdickt; diese verdickte Stelle hält bei noch weiterer Differenzierung den Farbstoff am längsten fest. An der Spitze des Perforatoriums vermag ich eine leichte knopfförmige Terdickung wahrzunehmen.

Am hinteren Umfang des Kopfes sieht man, wie Retzius geschildert bat, in der Seitenansicht zwei oder auch drei Kügelchen (siehe meine Fig. 1 a-d), welche sich (Retzius) durch Behandlung mit Osmiumsäure und Fuchsin (mit nachfolgendem Einlegen in essigsaures Kali) intensiv rot färben lassen. Hat man den Spermienkopf in der Ansicht von hinten vor sich (Fig. $1 \mathrm{e}-\mathrm{f}$ ), so erkennt man, dass im ganzen fünf solcher Kügelchen vorhanden sind, welche um den Ansatz des Schwanzes herum in einem regelmässigen Fünfeck liegen. Ich selbst erhielt sie bei Anwendung der Altmannschen Methode ebenfalls intensiv rot gefärbt; sie geben aber den Farbstoff bei diesem Verfahren sehr leicht wieder ab und erscheinen dann ganz durchsichtig und bell. Retzius hat äbnliche Kügelchen, deren Anzahl bald 4 , bald 5 , bald 8 beträgt, noch bei zahlreichen anderen Mollusken (Lamellibranchiern) und besonders bei Würmern (Polychaeten) aufgefunden.

Was nun die Deutung dieser Kügelchen anlangt, so sind sie bereits von Retzius (1904) als Homologa des Nebenkerns von v. la Valette St. Ge orge, des "Mitochondrienkörpers" von mir (1900) angesprochen worden. Dass diese Deutung zutreffend ist, ergibt sich in erster linie aus den spermatogenetischen Beobachtungen, welche M. v. Brun n (1884) und ich (1900) bei Paludina gemacht haben. In denjenigen Spermatiden von Paludina, aus welchen die eupyrenen Spermien entstehen, liegen, wie v. Brunn gezeigt hat, an der Hinterseite des sich ent- 
wickelnden Kopfes vier Kügelchen, welche „die Ecken eines winzigen Quadrats bilden, aus dessen Mitte der (Schwanz)faden hervortritt". Bei Paludina persistieren sie nicht als solche, sondern schliessen sich im Lauf der Entwicklung eng an den Schwanzfaden an (wobei sie sich mit ihren Wänden aneinander legen und verschmelzen) und strecken sich dann zu immer dünner werdenden Röhren in die Länge. Schliesslich sind sie in eine zylindrische, auf dem Querschnitt viergeteilte Umhüllung des sogenannten Mittelstücks des Schwanzfadens umgewandelt. Von diesen Kügelchen habe ich (1900) zeigen können, dass sie Derivate von Mitochondrien oder Plastochondrien und also Homologa des "Nebenkerns" bei Insekten sind, welcher nach meiner Feststellung gleichfalls plastosomatischer Natur ist. Die Kügelchen bei Mytilus sind nun zweifellos mit denjenigen bei Paludina identisch und können daher, wie es von Seiten Retzius' geschieht, in ihrer Gesamtheit als "Nebenkernorgan" bezeichnet werden.

Von dem Schwanz der Mytilusspermien sagt Retzius, dass er verbältnismässig kurz und mit einem Endstück versehen ist, "welches doppelt so lang ist als der eigentliche Kopf und auch länger als dieser zusammen mit seinem Perforatorium".

\section{Die reifen Eier.}

0. Hertwig, welcher 1877 bei Mytilus den Vorgang der Richtungskörperbildung .studiert hat, beschreibt, dass die frisch gelegten Eier sich auf dem Stadium der ersten Richtungsspindel befinden und dass sie von einer "festen, doppelt konturierten und glatt aufliegenden Membran" umschlossen werden. Von dem Protoplasma der Eier sagt er, dass es im lebenden Zustand ndurch kleine glänzende Körnchen in hohem Grade getrübt" erscheint. Schnitte von Eiern, welche nach der Altmannschen Methode behandelt worden sind, zeigen nun, dass zwei verschiedene Sorten von Körnchen existieren (Fig. 2). Ein Teil der Körnchen werden durch Osmiumsäure geschwärzt, stellen also Fett- oder Dotterkügelchen dar. Die übrigen erweisen sich durch ihre Farbungsreaktionen als Plastochondrien; sie besitzen meistens in einem und demselben $\mathrm{Ei}$ ein etwas verschiedenes Kaliber; die grössten erreichen beinahe die Grösse der Dotterkügelchen. Dotterkügelchen und Plastochondrien finden sich im allgemeinen 
bunt durcheinander gemengt; jedoch bildet die eine oder andere Sorte von Kügelchen vielfach besondere Anhäufungen.

Schon 0. Hertwig hat ferner im Mytilusei ein , $v 0$ der übrigen Dottersubstanz verschiedenes" kugeliges Gebilde wahrgenommen, „das in der Grösse von $3 \mu$ zu $\check{~} \mu$ variiert." „Man kann schwanken“, sagt $\mathrm{O}$. Hertwig, „ob es ein besonders beschaffenes Dotterelement oder ein aus Kernsubstanz bestehender Teil ist. Da indessen das Kügelchen einige Zeit nach der Befruchtung verschwunden ist und die Befunde auffallend an die bei Asteracanthion und Nephelis erhaltenen erinnern, so glaube ich mich für das letztere entscheiden zu müssen." 0 . Hertwig, welcher ausschliesslich Totalpräparate von Mytiluseiern untersucht hat, nahm an, dass es sich um einen in beständiger $\mathrm{Ab}$ nahme begriffenen kugelförmigen Rest des Keimflecks" handelt. Bei Schnittuntersuchung kann man nun aber leicht feststellen, dass vielmehr die erste der beiden Alternativen, zwischen welchen o. Hertwig geschwankt hat, zutreffend ist: es handelt sich um ein "besonders beschaffenes Dotterelement", und zwar um einen sogenannten "Dotterkern", wie wir ihn zuerst im Spinnenei besonders durch Balbiani kennen gelernt haben, bestehend aus einem zentralen protoplasmatischen Körperchen, welches in der Regel Plastochondrien und mitunter daneben auch noch Dotterkügelchen einschliesst, und umgebenden konzentrischen Lamellen, welche, dachziegelartig zusammengefügt, eine kugelige Kapsel bilden. Es kommt jedoch in zahlreichen Fällen vor, dass die Kapsel das zentrale Kügelchen nur einseitig umgibt, indem die konzentrischen Lamellen auf eine Seite desselben beschränkt sind (Fig. 2).

Auffallend ist, dass im Mytilusei nicht selten mehrere solcher Dotterkerne vorkommen. O. Hertwig erwähnt schon, dass das von ihm aufgefundene Gebilde "zuweilen auch in zwei Halften geteilt auftritt". Ich selbst habe einigemal drei und in einem Fall sogar vier solcher Dotterkerne gezăhlt.

\section{Die Befruchtung.}

Bei einer Portion Eier, welche ich $2^{1 / 2}$ Minuten nach der Besamung fixiert hatte, waren die Spermienköpfe zum Teil noch im Eindringen begriffen (Fig. 3), zum Teil aber schon völlig eingedrungen, jedoch noch unmittelbar unter der Eimembran gelegen 
(Fig. 4). Die Eimembran weist an der Eintrittsstelle des Spermiums ein rundes, scharf umgrenztes Loch auf, welches etwas weiter ist, als für den Durchtritt des Kopfes notwendig erscheint. Die Rander des Loches sind in den meisten Fallen etwas nach aussen umgebogen. Merkwürdig ist, dass das Perforatorium nicht nur bei den völlig aufgenommenen Köpfen, sondern auch schon bei den noch im Eintritt begriffenen spurlos geschwunden ist; seine Substanz muss sich also sehr rasch auflösen. Dagegen sind am hinteren Umfang des durch Hämalaun blau gefärbten Kopfes in Seitenansicht $z$ wei oder drei Kügelchen des "Nebenkernorgans" deutlich zu erkennen.

Indem der Kopf mit dem ihm anhaftenden Nebenkernorgan tiefer in das Ei eindringt, kommt er zwischen den geschwarrzten Dotterkügelchen und Eiplastochondrien zu liegen und wird nun besonders durch die erstèren den Blicken entzogen. Um ihn auffinden zu können, empfiehlt es sich, wie gesagt, die geschwärzten Kügelchen vorher durch Behandlung der Schnitte mit Terpentin zu entfernen, wie dies bei Fig. $\overline{0}-8$ geschehen ist. Fig. 5 und 6 sind nach Eiern gezeichnet, welche $4 \frac{1}{2}$ Minuten nach Zusatz des Spermas fixiert worden sind. In Fig. 5 und 8 hat sich von der Oberfläche der doppelt konturierten Eimembran eine "Dotterhaut" abgehoben; in Fig. 6 und 7 ist sie entweder noch nicht gebildet oder liegt der Eimembran noch so dicht an, dass sie nicht zu erkennen ist.

Der eingedrungene Kopf führt weiterhin eine Drehung aus, wie sie schon bei zahlreichen Tieren beobachtet wurde, in der Weise, dass er das vordere Ende gegen die Peripherie, das hintere gegen den Mittelpunkt des Eies kehrt: Fig. 7 und 8, nach Eiern, welche 6 Minuten nach der Befruchtung fixiert sind. In Fig. 7 liegt der Kopf genau rechts vom Eintrittsloch, aus welchem der Schwanzfaden hervorragt. Bei Fig. 8 ist das Eintrittsloch nicht auf dem Schnitt getroffen. In Ietzterer Figur haben die Kügelchen des „Nebenkernorgans" sich bereits von der Hinterseite des Spermienkopfes abgelöst. Nachdem sie sich weiter von ihm entfernt haben, besteht keine Möglichkeit mehr, sie von gleichgrossen Eiplastochondrien za unterscheiden. Damit ist der weiteren Verfolgung der männlichen plastosomatischen Substanz (wenigstens mit Hilfe der von mir angewandten Methode) im Mytilusei ein Ziel gesetzt. 
Immerhin ist es gelungen, an einem neuen Objekt den Nachweis zu erbringen, dass ausser dem Kern auch die Plastosomen der Samenzelle als geformte Elemente in das $\mathrm{Ei}$ eintreten. Dass sie dies allgemein tun, lassst sich allerdings, wie Duesberg (1912, S. 841) bereits bemerkt hat, überhaupt nicht im mindesten bezweifeln. Zunăchst konnte überall, wo die Bildung des Spermiums mit den geeigneten Methoden untersucht wurde, gezeigt werden, dass sämtliche Plastosomen der Spermatide dabei Verwendung finden. Wir wissen ferner, dass in zahlreichen Fällen die ganzen Spermien einschliesslich des Schwanzes in das Ei aufgenommen werden. ${ }^{1}$ ) ,Andererseits “, sagt Du e sberg, $n^{z}$ eigen die Untersuchungen über den Aufbau des reifen Spermatozoids, dass es in vielen Fällen genügt, wenn ein sehr kleiner Teil des Schwanzes in das $\mathrm{Ei}$ eindringt und in einigen Fällen dieses Eindringen [des Schwanzes] 'gar nicht nötig ist, um die Plastosomen des Spermatozoids in das Ei zu bringen."

Es kann allerdings zunächst befremden, dass die Plastosomen des Spermiums denjenigen des Eies in vielen Fällen z. B. bei Mytilus und Phallusia so ausserordentlich an Masse nachstehen ${ }^{2}$ ); aber ein Argument gegen ihre Mitwirkung bei der Vererbung lässt sich, wie ich schon mebrfach bemerkt habe, daraus nicht berleiten. ${ }^{3}$ ) Die Stammzellen des Spermiums und der Eizelle, die Spermatogonien und Oogonien, sind bei vielen Tieren nicht nur an Grösse, sondern auch in bezug auf den Bau

1) Man kann es heute sogar für wahrscheinlich halten, dass alle Angaben, nach welchen Schwanzteile abgeworfen werden, auf Irrtum beruhen.

$\Rightarrow$ Bei Ascaris und Filaria ist dies weniger der Fall.

3) Man vergleiche auch W. Pf effer, Pflanzenphysiologie, II. Auth.s 1897, Bd. 1, S. 46: „Da mit dem Samenfaden (wie es scheint, in allen Fällen) bei der Befruchtung der Eizelle auch Zytoplasma zugeführt wird, so kann schon dieserhalb aus den bezüglichen Erfahrungen die Alleinherrschaft des Kerns mit Recht nicht gefolgert werden .... Weiter heisst es S. 47: „In diesen Fragen ist aus der relativ ansehnlichen Grösse des Kernes in embryonalen Zellen ein entscheidendes Argument nicht abzuleiten, so beachtenswert und bedeutungsvoll diese Tatsache auch ist. Denn von der Körpermasse hängt doch nicht die Bedeutung eines Menschen im Gemeinwesen $a b$, und die Bakterien demonstrieren sehr schön, wie eine winzige lebendige Masse, indem sie zu intensiver Vermehrung befähigt ist, die gewaltigsten Leistungen zu vollbringen and selbst die grössten Organismen zu vernichten vermag. Zudem können gewaltige Reizerfolge durch unglaublich geringe Mengen ausgelöst werden." 
von Kern und Zytoplasma einander so völlig gleich, dass sie sich überhaupt nicht unterscheiden lassen. Die Oogonie pflegt mit ihrem Übertritt in die Wachstumsperiode eine gewaltige Vergrösserung zu erfahren, an welcher nicht nur das Zytoplasma, sondern auch der Kern Teil hat, so dass das "Keimblaschen" den Kern der homologen männlichen Zelle, des Spermatozyten erster Ordnung, in vielen Fälen um das hundertfache und mehr übertrifft. Nichtsdestoweniger erweisen sich die Kerne der beiden kopulierenden Geschlechtszellen als „aquivalent“. Was nun die Plastosomen anlangt, so könnte die im frisch besamten Ei vorhandene Ungleichbeit in der Zahl der männlichen und weiblichen Plastosomen durch ein starkes Wachstum der ersteren beseitigt werden. Dabei ist zu betonen, dass ein solcher Ausgleich sich keineswegs bis zum Beginn der ersten Furchung (Auftreten der Zelleibsteilung) zu vollziehen braucht: in den Furchungszellen ist noch Zeit genug dafür. Die Befruchtung vollendet sich meines Erachtens vielfach erst im Lauf der Keimbildung.

Solange man glauben durfte, dass sämtliche erblichen Eigenschaften im Kern vereinigt sind, war die Annahme notwendig, dass sie von diesem auf das Zytoplasma übertragen werden. Wenn bei Kreuzung einer rot und einer weiss blühenden Pflanze die Blumen des Bastards eine intermediare blassrote Färbung aufweisen, so mussten wir uns früher vorstellen (De Vries 1889), dass die Chromatophoren ihre Eigenschaften vom Kern mitgeteilt bekommen haben. Man zog zur Erklärung eine dynamische oder enzymatische Wirkung des Kerns auf das Zytoplasma heran oder griff zu der Hypothese, dass die im Kern enthaltenen stofflichen Träger der erblichen Anlagen (Pangene, De Vries) rom Kern an das Zytoplasma abgegeben werden. Mit der Erkenntnis, dass neben dem Kern auch die Plastosomen bei der Befruchtung mitwirken, sind alle diese Annahmen überflüssig geworden. Die Plastosomen sind die Vererbungsträger des Zytoplasmas; sie stellen nach der Anschauung, welche ich mir von 1907 an auf Grund meiner Beobachtungen gebildet babe, eine primitive (indifferente, neutrale) Substanz dar, welche sich selbst im Lauf der Ontogenese in die verschiedensten Differenzierungen umwandelt, wobei sie die elterlichen Eigenschaften in die Erscheinung treten lässt. Wenn in dem oben angeführten Beispiel die Blüten der Bastardpflanze blassrot sind, so erklärt sich dies 
meiner Meinung nach daher, dass im Lauf der Befruchtung männliche und weibliche Plastosomen sich miteinander vereinigt haben und dass die Chromatophoren der Pflanzen, wie neuere Untersuchungen gezeigt haben, (ebenso wie die Pigmentkörner der Tiere) Umwandlungsprodukte von Plastosomen sind. ${ }^{1}$ )

In einer früheren Arbeit (1908) habe ich die Frage untersucht, inwieweit auf die Plastosomen die Vorstellung passt, welche $\mathrm{Naegeli}$ sich von der ausseren Erscheinung und der Struktur seines Idioplasmas gebildet hat, und bin $\mathrm{zu}$ dem Resultat gelangt, dass die Plastosomen eine geeignete Grundlage für die Naegelische Theorie innerhalb des Zytoplasmas abgeben. Jedoch fand ich, dass die Anschauungen, welche von Na egeli mit Bezug auf die spezifische Wirksamkeit des Idioplasmas entwickelt worden sind, durch meine Beobachtungen über das Verhalten der Plastosomen bei der „Entfaltung der Anlagen" keine Bestätigung erhalten. Ich kam nämlich zu dem Ergebnis, dass die verschiedenen Differenzierungsprodukte der Zellen nicht, wie es die Theorie $\mathrm{Naegelis}$ verlangen würde, durch Einwirkung der Plastosomen (bezw. der den Plastosomen innewohnenden Molekularkrafte) auf das umgebende Zytoplasma hervorgebracht werden; sie entstehen vielmehr nach meiner Ansicht, wie ich schon eben bemerkt habe, aus den Plastosomen selbst a uf dem Wege direkter Metamorphose, welche nach den verschiedensten Richtungen vor sich geht.

In dieser Beziehung stimmt mit meiner Auffassung in viel höherem Grade die Lehre überein, zu welcher Galton durch das Studium der Erblichkeitsgesetze geführt worden ist. Johannsen, dessen „Elementen der exakten Erblichkeitslehre"

1) Nach Strasburger (1908) soll ja allerdings bei der Befruchtung der Phanerogamen ein "nackter Spermakern" in das Bi hineinschlüpfen; ein Erguss von zytoplasmatischem Pollenschlauchinhalt in das $\mathrm{Ei}$ ist nach Strasburger in keinem Fall bisher beobachtet worden. Ich habe aber hierzu schon 1908, S. 859 folgendes bemerkt: „Da das Zytoplasma des Pollenschlauchs den Spermakern, wie S tra s bu rger selbst (1908, S. 40) sagt, an seinen Bestimmungsort befördert, so lässt es sich meines Erachtens nicht ausschliessen, dass etwas davon mit in das Ei hineingelangt. Ferner ist aber die Möglichkeit nicht abzuweisen, dass schon ein einziges winziges Mitochondrium genügen könnte, um die Eigenschaften des väterlichen Zytoplasmas auf dasjenige des Eies zu übertragen." 
(erste dentsche Ausgabe 1909, S. 478 u. folg.) ich meine Kenntnis dieser Theorie verdanke, gibt davon folgende Darstellung. Gal t on (1875), führt er aus, nennt dasjenige im befruchteten $\mathrm{Ei}$, was für die Vererbungserscheinungen massgebend ist, den Stirp (aus dem lateinischen stirps, Stamm), ein Wort, welches sich auch durch die häufig benutzten Ausdrücke Idioplasma (Naeg eli 1854) und Keimplasma (Weismann 1885) ersetzen lässt. Fr denkt sich, dass die Sexualzellen (und ebenso die embryonalen Gewebe) reich an Stirp sind und dass dieser in den Sexualzellen direkt von der einen Generation zur folgenden weitergefübrt wird, ohne in den speziellen persönlichen Entwicklungsgang des einzelnen Individuums hineingezogen zu werden, wahrend die spezialisierten Körperzellen im Lauf ihrer Entwicklung das ibnen überlieferte "Keimplasma" grösstenteils „verbrauchen. " ${ }^{1}$ ) Die Sexualzellen der nacheinander folgenden Generationen bilden ein Kontinuum, eine Fortsetzungsreihe; und es ist sehr deutlich, dass dadurch der Stirp das eigentlich bleibende, das eigentlich "feste " der betreffenden Rasse bildet. „Die individuellen Körper, die Einzelpersonen, sind - mit einem nicht ganz adäquaten Bild übrigens - vergänglichen Blätern oder Trieben an einem unsichtbaren Wurzelstock ähnlich; der „Wurzelstock“ wird von den Blättern und Trieben ernăhrt, diese aber manifestieren nur, was im "Wurzelstock" gegeben ist aber in höchst wechselnder Art je nach den Schwankungen der Lebenslagefaktoren".

Diese Lehre kann nach Johannsen (1909, S. 480) in einfachster Weise durch folgendes Schema illustriert werden:

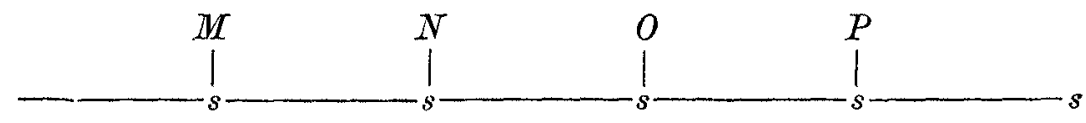

„Hier bezeichnen die Buchstaben M-P vier Generationen von Individuen, während s den Stirp (das Keimplasma) der Gameten bezeichnet, welcher alle Generationen zur einheitlichen Entwicklungsreihe verbindet. Die langen Striche des Schemas deuten das Freiwerden, das Ausscheiden von Gameten, z. B. eines Eies, an; die kurzen Striche bezeichnen die Entwicklung des betreffenden Individuums aus der grundlegenden Zygote."

1) Man vergleiche hierzu $J_{0}$ hannsen, Elemente der exakten Erblichkeitslehre, zweite deutsche Ausgabe, 1913, S. 408. 
Weiter (S. 487) unternimmt es Johannsen, das Galtonsche Schema an die Mendelsche Regel zu adaptieren, wobei er die beiden zur Kreuzung verwendeten rassenreinen Individuen (die "homozygotischen P-Formen") mit AA und aa bezeichnet; das Schema z. B. der AA-Form würde dann folgendermaßen auszufübren sein :

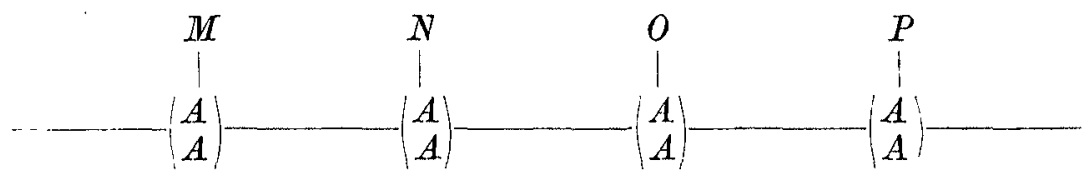

Das obere A bezeichnet die "genotypische" Beschaffenheit der Eizelle, das untere diejenige der Samenzelle. Hier sind beide genotypisch gleich und könnten darum auch mit $s$ (wie vorher) markiert werden.

„Die Berücksichtigung der „Personen“, M, N usw., ist nun offenbar hier unnötig und macht das Schema für den weiteren Gebrauch nur schwerfällig. Halten wir uns allein an die genotypische Beschaffenheit der Gameten bezw. der Zygoten, dann können wir hier gleich das derart vereinfachte Schema der beiden P-Formen sowie des Bastardes beider darstellen. Mit $\mathrm{G}_{1}-\mathrm{G}_{4}$ sind die betreffenden vier Generationen markiert":
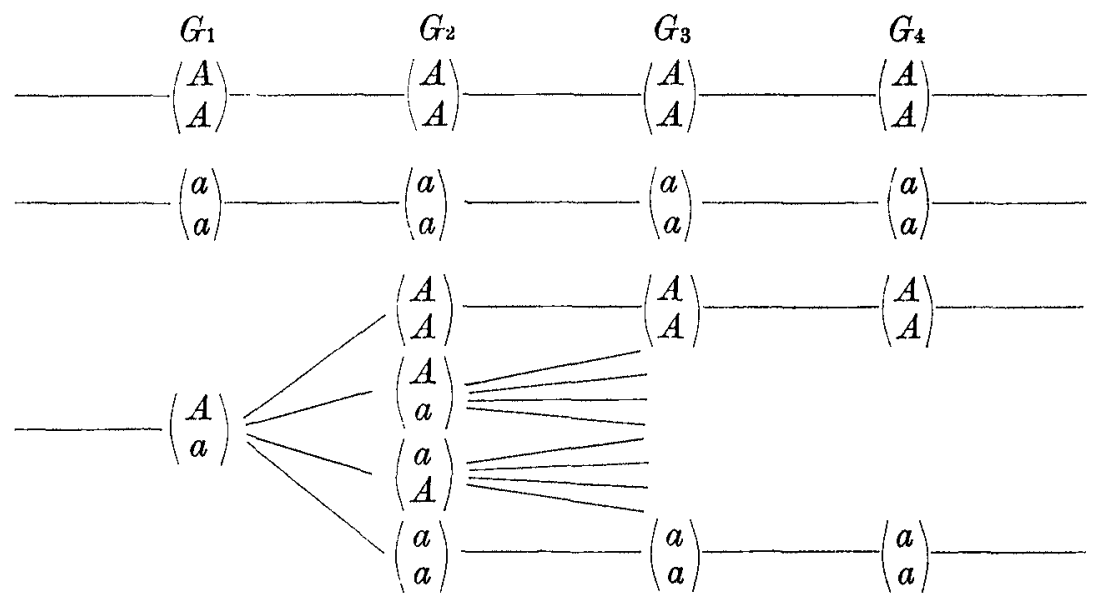

Dieses Bastardschema ist, wie Jobannsen sagt, „nichts ais eine graphische Transskription des einfachen Mendelschen 
Spaltungsschemas bei Selbstbefruchtung des Bastards $F_{1}{ }^{*}$ Um das Verhalten der Nachkommenschaft eines solchen Bastards zu erklaren, hat Mendel bekanntlich die Hypothese aufgestellt, dass die in $F_{1}$ zusammengebrachten Gene oder Erbeinheiten be i der Bildung der Sexualzellen wieder getrennt würden oder mit anderen Worten, dass jeder Bastard der $F_{1}$-Generation zweierlei Arten von Sexualzellen (zweierlei männliche und zweierlei weibliche) bilde. Die zytologische Grundlage der Spaltungsprozesse glaubt man vielfach in der sogenannten "Reduktionsteilung" gefunden $z u$ haben, welche Weismann, ohne Kenntnis des Mendelschen Vererbungsmodus, von theoretischen Erwägungen aus postuliert hatte. Ich darf dazu bemerken, dass ich schon 1902 konstatiert habe (vergl. auch Meves 1896), dass eine solche Reduktionsteilung als allgemeines Vorkommnis nicht existiert. Meine weiteren Untersuchungen, zusammen mit der Konfusion, welche bezüglich des "Reduktionsproblems" in der zytologischen Literatur von Anfang an geherrscht und immer stärker um sich gegriffen hat, haben die Überzeugung in mir immer mehr befestigt, dass die W e is man n sche Reduktionsteilung das Pbantasieprodukt bleiben wird, als welches sie entstanden ist.

Ferner scheint es mir aber keineswegs erforderlich, anzunehmen, dass die Spaltung und Neukombination der Gene oder Erbeinheiten schon bei der Bildung der Sexualzellen der $F_{1-}$ Generation vor sich geht. Vielmehr möchte ich mit Na geli (1884, S. 208) glauben, dass diese Erscheinungen in erster linie von dem Verhalten der bei der Selbstbefruchtung des Bastards $F_{1}$ (bezw. bei der Befruchtung innerhalb der Fi-Generation) zusammenkommenden Idioplasmen abhängig sind.

\section{Literaturverzeichnis.}

Balbiani, E. G., 1879: Leçons sur la génération des Vertébrés. Paris. v. Brunn, M., 1884: Untersuchungen über die doppelte Form der Samenkörper von Paludina vivipara. Arch. f. mikr. Anat., Bd. 23.

Duesberg, J., 1912: Plastosomen, „apparato reticolare interno ${ }^{*}$ und Chromidialapparat. Ergebn. d. Anat. n. Entwicklungsgesch.. Bd. 20.

Held, H., 1912: Über den Vorgang der Befruchtung bei Ascaris megalocephala. Ber. d. Kgl. sächs. Ges. d. Wiss., math.-phys. Kl. und Verhandl. d. anat. Ges, auf d. 26. Vers. in München. 
Hensen, V., 1881: Physiologie der Zeugung. Handbuch der Physiologie, Bd. 6, II. Teil.

Derselbe, 1885: Die Grundlagen der Vererbung nach dem gegenwärtigen Wissenskreis. Landwirtschaftl. Jahrb., Bd. 14.

Hertwig, 0., 1875: Beiträge zur Kenntnis der Bildung, Befruchtung und Teilung des tierischen Eies. I. Abh. Morpholog. Jahrb., Bd. 1.

Derselbe, 1877: Beiträge zur Kenntnis der Bildung, Befruchtung und Teilung des tierischen Eies. Dritter Teil. Morpholog. Jahrb., Bd. 4.

II eves, Fr., 1896: Über die Entwicklung der männlichen Geschlechtszellen von Salamandra maculosa. Arch. f. mikr. Anat., Bd. 48, 1897.

Derselbe, 1900: Über den von v. la Valette St. George entdeckten Nebenkern (Mitochondrienkörper) der Samenzellen. Arch. f. mikr. Anat., Bd. 56 .

Derselbe, 1902: Über oligopyrene und apyrene Spermien und über ihre Entstehung, nach Beobachtungen an Paludina und Pygaera. Arch. f. mikr. Anat., Bd. 61.

Derselbe, 1907: Über Mitochondrien bezw. Chondriokonten in den Zellen junger Embryonen. Anat. Anz., Bd. 31.

Derselbe, 1908: Die Chondriosomen als Träger erblicher Anlagen. Cytologische Studien am Hühnerembryo. Arch. f. mikr. Anat., Bd. 72.

Derselbe, 1911: Über die Beteiligung der Plastochondrien an der Befruchtung des Eies von Ascaris megalocephala. Arch. f. mikr. Anat., Bd. 76.

Derselbe, 1913: Über das Verhalten des plastosomatischen Bestandteiles des Spermiums bei der Befruchtung des Eies von Phallusia mammilata. Arch. f. mikr. Anat., Bd. 82, Abt. 2.

Derselbe, 1915: Über Mitwirkung der Plastosomen bei der Befruchtung des Eies von Filaria papillosa. Arch. f. mikr. Anat., Bd. 87, Abt. 2.

v. Na egeli, C., 1884: Mechanisch-physiologische Theorie der Abstammungslehre.

Retzius, G., 1904: Biologische Untersuchungen, N. F., Bd. 11.

Romeis, B., 1912: Beobachtungen über Degenerationserscheinungen von Chondriosomen. Nach Untersuchungen an nicht zur Befruchtung gelangten Spermien von Ascaris megalocephala. Arch. f. mikr. Anat., Bd. 80, Abt. 2.

Strasburger, E., 1908: Chromosomenzahlen, Plasmastrukturen, Vererbungsträger und Reduktionsteilung. Jahrb. $f$. wiss. Bot., Bd. 44.

Van Beneden, E., 1883: Recherches sur la maturation de l'oeuf, la fecondation et la division cellulaire. Archives de Biologie, vol. 4.

De Vries, H., 1889: Intrazellulare Pangenesis. Jena.

Z o ja, L. und R., 1891: Intorno ai plastiduli fucsinifoli (bioblasti dell' Altmann). Mem. Ist. Lomb. Sc. Lett., Milano, vol. 16. 
62 Friedrich Meves: Befruchtungsvorgang bei der Miesmuschel.

\section{Erklärung der Abbildungen auf Tafel V.}

Die Abbildungen der Tafel $V$ sind mit Zeiss' Apochromat $2 \mathrm{~mm}$ (Apertur 1,40) und Kompensationsokular 12 unter Benutzung des $A b$ b e schen Zeichenapparates bei Projektion auf Objekttischhöhe gezeichnet; und zwar sämtlich nach Präparaten, welche mit A Itmannschem Gemisch fixiert und mit Hämalaun und Fuchsin-Pikrinsäure gefärbt worden sind.

Fig. 1 a-d. Spermien von Mytilus in Seitenansicht. Näheres siehe Text.

Fig. 1e. Ansicht des Spermienkopfes mit den fünf Kügelchen des Nebenkernorgans von hinten.

Fig. 1f. Die fünf Kügelchen des Nebenkernorgans von hinten gesehen, mit einem nach unten links ziehenden Stück des Schwanzfadens.

Fig. 2. Reifes Mytilusei, Dotterkïgelchen geschwärzt, Plastochondrien rot gefärbt. Richtungsspindel und "Dotterkern". Näheres siehe Text.

Fig. 3-8. Teile von Schnitten durch befruchtete Mytiluseier. Bei Fig. 5-8 sind die geschwärzten Dotterkügelchen durch Terpentin weggelöst.

Fig. 3 und $4: 2^{1 / 2}$ Min., Fig. 5 und $6: 4^{1 / 2}$ Min., Fig. 7 und 8: 6 Min. nach der Besamung fixiert. Im übrigen siehe Text. 


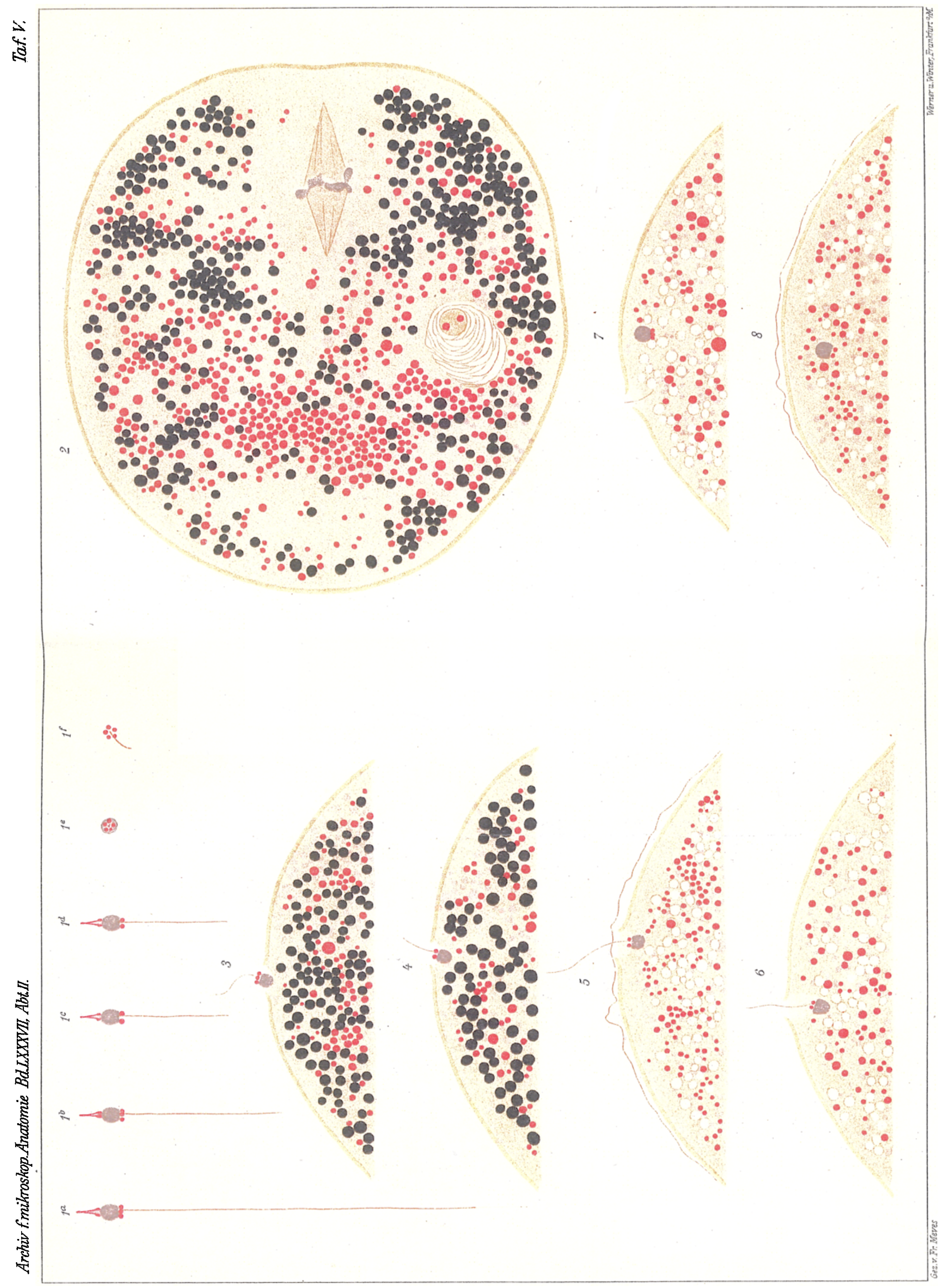

